\title{
The early career researcher's toolkit: translating tissue engineering, regenerative medicine and cell therapy products
}

\author{
Qasim A. Rafiq ${ }^{*+1,2}$, Ilida Ortega*3, Stuart I. Jenkins*4, Samantha L. Wilson*5, Asha K. \\ Patel $^{* 6,7}$, Amanda Barnes ${ }^{* 8}$, Christopher F. Adams*4, Derfogail Delcassian*9,10 and \\ David Smith*1,11
}

${ }^{1}$ Centre for Biological Engineering, Wolfson School of Mechanical and Manufacturing Engineering, Loughborough University, Leicestershire, LE11 3TU, United Kingdom.

${ }^{2}$ Aston Medical Research Institute, School of Life and Health Sciences, Aston University, Aston Triangle, Birmingham, B4 7ET, United Kingdom.

${ }^{3}$ Bioengineering and Health Technologies Group, The School of Clinical Dentistry, University of Sheffield, S10 2TA, United Kingdom.

${ }^{4}$ Institute for Science and Technology in Medicine, Keele University, Staffordshire, ST5 5BG, United Kingdom.

${ }^{5}$ Academic Ophthalmology, Division of Clincial Neuroscience, Queen's Medical Centre Campus, University of Nottingham, NG7 2UH, United Kingdom.

${ }^{6}$ Wolfson Centre for Stem Cells, Tissue Engineering and Modeling, University of Nottingham, Nottingham, NG7 2RD, United Kingdom.

${ }^{7}$ David H. Koch Institute for Integrative Cancer Research, Massachusetts Institute of Technology, Cambridge, Massachusetts 02139, United States.

${ }^{8}$ Biomedical Tissue Research Group, University of York, YO10 5DD, United Kingdom.

${ }^{9}$ Department of Materials, Imperial College London, Exhibition Road, London SW7 2AZ, United Kingdom.

${ }^{10}$ Drug Delivery and Tissue Engineering, Centre for Biological Sciences, School of Pharmacy, University of Nottingham, NG7 2UH, United Kingdom.

${ }^{11}$ PCT, a Caladrius company, 4 Pearl Court, Suite C, Allendale, NJ 07401, United States.

* All authors contributed equally to this work.

$\dagger$ Author for correspondence. (Tel: +44-121 204 4895; E-mail: q.rafiq@aston.ac.uk) 


\begin{abstract}
Although the importance of translation for the development of tissue engineering, regenerative medicine and cell-based therapies is widely recognized, the process of translation is less well understood. This is particularly the case among some early career researchers who may not appreciate the intricacies of translational research or make decisions early in development which later hinders effective translation. Based on our own research and experiences as early career researchers involved in tissue engineering and regenerative medicine translation, we discuss common pitfalls associated with translational research, providing practical solutions and important considerations which will aid process and product development. Suggestions range from effective project management, consideration of key manufacturing, clinical and regulatory matters and means of exploiting research for successful commercialization.
\end{abstract}

\title{
Keywords
}

Translation, Tissue Engineering, Regenerative Medicine, Cell Therapy, Stem Cell, Early Career Researcher, Manufacture, Clinical and Preclinical Models, Translational Research 


\section{Introduction}

The development of reimbursable cell therapies, tissue engineered constructs and regenerative medicines poses significant technical and commercial challenges. However, increasing numbers of therapies are being approved by international regulatory bodies, with initial therapies now gaining reimbursement. To accelerate the development of promising products in the tissue engineering and regenerative medicine (TERM) field, translational research needs to underpin and drive the development process. Translational research, as we have defined it, is that which takes science from the bench by addressing specific technological challenges subsequently enabling the delivery of healthcare and economic benefits. As researchers at the forefront of this nascent industry, we have come to realize that the process of translation can be intricate, exceptionally resource consuming and vulnerable to unexpected obstacles. What follows is a compilation of 'tips and tricks' from the perspective of early career researchers (ECRs), based on our own experience and research, which facilitate translation and will improve the chances of successful product development. While this is aimed at other ECRs, we believe the strategies contained herein will be of benefit to all translational researchers in the TERM field. 


\section{Manage the research effectively}

Perhaps the most important factor in the translation and development of a TERM therapy, or in any project, is its management. Without a clear and detailed research management plan, resources may be wasted and deliverables unmet. Outlined below are strategies to adopt in the day-to-day running of a translational research project.

\subsection{Utilise project management principles}

The success of a project is ultimately based upon diligent planning and execution rather than the results achieved; 'negative' results can be just as informative as 'positive'. Dividing projects into discrete work packages with clear milestones and deliverables enables resource allocation (Section 1.2) and helps in identifying potential bottlenecks and high risk elements for which mitigation/contingencies can be planned, for example, obtaining ethical approval for human-derived materials, or licensing for in vivo research. Likewise, key equipment may fail, therefore contingencies should be established in the form of service agreements, maintenance plans and where possible, alternative providers. Failure to plan ahead in these areas can significantly hinder or even halt progress of a project. Risk must be managed by anticipating it - risk registers have value.

Comprehensive project planning is essential for successful translation. The project plan is best represented by a Gantt chart with a realistic level of detail that can be followed by all stakeholders with a personal guideline of one deliverable per research associate per quarter. The plan should be prepared and/or reviewed by all stakeholders to ensure all understand and believe in it. Translational research is often a high-risk activity and projects do not always 
progress as planned. It is therefore good discipline as part of the project plan to establish phase gates where the conditions for proceeding are clear. If unavoidable delays occur in one work package, identify other packages or, at a minimum, sections of a package, which could be brought forward. Milestones provide the opportunity to adapt and reassess the project as appropriate, and must have associated contingencies, so that if milestones are not achieved (e.g. processes are not reproducible, or outcomes predicted from in vitro data are not observed in vivo), pre-identified alternatives are investigated. In some situations, it may be necessary to terminate projects and/or work packages because they are no longer a good use of resources/effort. Where alternative directions for the research are pursued, the reasons for doing so must be strategic; the traditional academic approach of 'I did this because it was more interesting' is not acceptable in a translational setting.

Understanding project management tools as used in large companies for large projects is an important part of training, professional development and understanding the environment. It is worth noting, however, that for smaller projects in an academic setting, their full implementation can be overkill and recognising how to tailor these tools to achieve the desired outcome is equally important.

\subsection{Prepare for day-to-day as well as long-term working}

Effective project management should also account for the resources required for each experiment. Prior to experimentation, ensure sufficient quantities of consumables/reagents are in stock, and note which require advance preparation (e.g. thawing, sterilizing). Reserve necessary equipment and ensure their working operational status. When laboratory space, 
equipment and consumables are communal, it is good practice to appoint specific people to monitor stocks and set minimum threshold levels for key items. Similarly, establish procedures to ensure critical quality and safety activities are performed in line with good laboratory practice (GLP, discussed further in Section 4.2), for example, frequency of safety cabinet and incubator cleaning. Alongside this, having standard operating procedures (SOPs) with detailed methodologies helps maintain comparability between experiments and can serve as checklists, thereby maximising efficiency and eliminating errors.

\subsection{Create an efficient data trail}

Vast amounts of data can be produced from experimental procedures and analysis which require careful management. Whether on computers or in laboratory notebooks, do not simply name data/files by date or generic titles. This can cause difficulties in tracing specific data, potentially critical for intellectual property (IP) claims. Each experiment/analysis should receive a unique ID linking the scientist, paperwork and the electronic files containing both raw and analysed data. Electronic storage of completed notebooks is common industry practise, and where possible should be emulated in academia. Furthermore, and in addition to their requirements for IP, many publicly-funded research bodies have specific requirements for data management. Indeed, in light of the recent stimulus-triggered acquisition of pluripotency (STAP) controversy, data management is under greater scrutiny and it is likely that we may witness increased levels of internal auditing and data verification [1]. 


\section{Ensure the data are scientifically robust}

Misleading 'data' can be very costly [1,2]. Data robustness should be ensured through rigorous experimental design, appropriate use of statistical methods and familiarity with principles of key technologies.

\subsection{Invest time in planning and designing experiments}

Heed the words of G.J. Quarderer (Dow Chemical Co.), "Four to six weeks in the lab can save you an hour in the library" [3]. TERM experiments are expensive, can take weeks, even years, to complete, and involve complex biological systems. Yet in an effort to start collecting data, researchers sometimes rush into experimental work without due diligence and planning, often resulting in poorly designed experiments.

Systematic methods such as factorial design of experiments (DOE) are valuable tools that can be used to design statistically relevant experiments, identify key variables and enhance both process and product understanding [4-7]. This places the emphasis on extensive planning and preparation prior to undertaking experimental work, and requires careful data analysis upon completion. The vast amount of useful data generated with minimal resource utilisation from a thorough DOE is far greater than the 'one factor at a time' (OFAT) approach, and likely to provide more value for TERM applications given the ability to identify relationships between factors at multiple levels. By taking the time upfront to plan, one can ensure that experiments are designed such that they are statistically valid with appropriate statistical tests employed to interrogate the data. No statistical 'ingenuity', however, can turn poor raw data into meaningful information.

\subsection{Source raw materials carefully}


Maintaining supply of within-specification raw materials is essential for consistent manufacture, but may prove particularly problematic for biological materials. Reliance on animal-derived biological materials should be eliminated where possible, but without adequate replacements, many processes still rely on them (e.g. foetal bovine serum, FBS). Many constituents of FBS are undefined and even measurable elements vary between lots. With this in mind, and where applicable, it is good practice to test multiple raw material lots in order to identify critical material attributes (CMAs) relevant to desired outcomes, evaluate inherent variability in these, and establish limits/thresholds to form the basis of raw material specifications. The CMAs help guide towards defining the critical quality attributes (CQAs) of the product - product characteristics that must be within specified limits to ensure safety, identity, purity and potency, e.g. cell marker expression or proliferative capacity [8-10]. Subsequently, as raw material lots near depletion or expiry, tests should be performed to identify new lots that satisfy the CMA requirements, and in turn ensure the product CQAs are still met. Variation is inherent to biological materials but retaining as much control as possible over measurable factors can lead to more consistent, reproducible processes [9].

The practice of reserving and buying specific lots applies to cell culture reagents generally, particularly those exhibiting biological variability such as Matrigel ${ }^{\mathrm{TM}}$. For items which are manufactured by multiple suppliers, such as basal media and amino acid supplements, it is good practice to repeat-purchase from established suppliers [11]. Whilst this increases supplychain risk, benefits arise in securing a consistently manufactured raw material and in developing long-term relationships with suppliers which may lead to discounted prices and preferential choice of stock. The selection process for supply of key reagents has to be rigorous and stringent (including due diligence of the supplier), and if it is necessary to switch suppliers, 
it is imperative to determine whether potential new materials generate products that meet the CQAs.

\subsection{Understand the principles behind technologies}

Key instruments, such as fluorescence-based systems, have become 'black-boxes', requiring minimal user intervention and are commonly managed by a core facility. While this increases efficiency and accessibility, users can fail to appreciate the limitations or potential of the technology, including safety/quality critical protocols, due to a lack of understanding of the underlying principles on which the technology is based. Greater understanding of these principles will enrich data collection, facilitate reliable data interpretation, and enable the development of customisable applications. All of this can be achieved by working with experienced users/technicians.

An example of this is flow cytometry (FC), which involves analysis of cell size, granularity and marker expression, whilst a similar technology, fluorescence activated cell sorting (FACS), sorts cells on these bases. TERM studies that employ FC often use only single colour antibody staining [12]. While effective in obtaining reproducible data, this method is cumbersome, requires large quantities of sample for analysis and, more critically, yields less information in comparison to multiparameter FC. User understanding should therefore include identification of appropriate isotype controls, instrument-relevant fluorophore combinations to allow for multiparameter analysis alongside accurate compensation and 'gating' settings to distinguish cell types [13]. 
Understanding such principles can also improve device operation, and hence data reliability. For example, FACS does not sort cells, but rather charged droplets containing the cells; understanding this should promote diligence in charging plate maintenance, applying the correct charge and accurate positioning of the break-off point. Also, FC/FACS instruments are sensitive to atmospheric and temperature changes. Therefore, they require daily calibration, which should be factored into process design for clinical production. 


\section{Ensure the work has clinical relevance}

Although clinical translation can seem a distant prospect from some regions of the TERM landscape, increased impact, utility and adoption of the technology can be achieved by considering its clinical relevance.

\subsection{Align the research with appropriate 'clinical pull'}

Direct the research towards 'clinical pull' factors (Textbox1), which can be broadly split into three categories, namely, benefit to public health, reimbursement available to recoup investment and utility for clinicians. Indeed, addressing unmet clinical needs will likely be crucial for the translational success of regenerative therapies [14, 15]. Following benefits to public health, likelihood of reimbursement will be, in part, determined by predicted savings for healthcare providers/insurers based on current lifetime costs of treatment and care. Such lifetime savings for chronic conditions are currently attractive opportunities for regenerative medicines [15]. For example, although treatments for spinal cord injury are potentially expensive, they may attract substantial reimbursement due to savings on healthcare personnel time, through increased patient autonomy. On the other hand, acellular products may warrant limited reimbursement per item, but could provide advantages in terms of manufacturing costs, logistics and a simpler regulatory pathway, favouring a commercial plan to treat large numbers of patients [7]. The counterpart to clinical pull, 'technology push', can provide new engineering tools (e.g. CRISPR [16]) with strong IP that is attractive to investors, but without alignment to clinical pull there is little prospect of successful translation [14, 17-19].

\section{[Textbox 1]}

Clinical pull - Value to patients, clinicians, healthcare providers/insurers and industry can be broadly quantified by assessing: 
- Demand for treatments (current patients; new patients/year; treatments/patient)

- Utility for clinicians (likelihood of technology uptake in the clinic)

- Potential quality of life improvements (e.g. as assessed by quality-adjusted life years [20-23])

- Lifetime savings versus costs of current care (number of procedures; expected lifetime with condition)

- Manufacturing costs and reimbursement per treatment (profit)

Often overlooked in terms of clinical pull is the need for enthusiasm amongst clinicians with respect to the utility of the technology, without which the product may never be adopted. Therefore, engagement with clinicians in order to assess their needs and wants is essential. Other benefits include feedback regarding therapeutic successes/failures, insights into the practicalities of administering therapies and clinical trial support/funding [24]. An example of this has been reported by researchers in the Moorfields Eye Hospital, with such arrangements providing competitive UK advantages [25].

\subsection{Broaden the applicability of the research: increase chances of success}

Can the research serve as a platform technology applicable to other conditions? Platform technologies can enhance the translational utility of the research, providing multiple chances of a 'hit', reducing risk and conferring greater commercial value [15]. For example, drug/cell delivery systems could be developed with variants designed to administer different payloads, potentially treating a wide variety of pathologies. Indeed, companies such as Organogenesis, Athersys and Mesoblast have products which are approved for multiple indications, and this approach increases the chance of commercial viability [15]. However, resources should be focussed on a limited number of candidates at first, leaving the others in reserve. 


\subsection{Use predictive and relevant disease/injury models}

Among the most influential statisticians of his generation [26], George E.P. Box wisely noted "all models are wrong, but some are useful" [27]. It is important to quickly assess the suitability of safety and efficacy assays to avoid wasting resources on models known to lack predictive value. Indeed, it is increasingly recognised that many in vitro and in vivo models lack predictive utility [28, 29], with vast resources wasted on clinical trials that fail in spite of impressive preclinical data. With this in mind however, substantial inertia must be overcome for flawed models to be abandoned. These limitations can be addressed by employing a robust range of models to identify weaknesses early on, reducing the likelihood of failure when facing the heterogeneity of human patients [30]. In particular, many clinical trials employ patient stratification, as safety and efficacy frequently vary with patient age, sex, co-morbidity, genetics etc [31]. Preclinical testing can be similarly stratified by separately analysing multiple strains/species/cell lines, and perhaps co-morbidity models. In the coming era of personalised medicine, such data could identify the patients most likely to benefit from specific therapies, and spare those likely to be harmed.

The problems with current preclinical testing offer rich opportunities for academia and industry, as TERM therapies will require new assays [32]. There are financial and ethical gains to be made by developing new models that address the 3Rs (replacement, reduction and refinement of animal models), with the ideal being the complete replacement of animal usage [33-35]. The greatest incentive to abandon unreliable systems will be the development of methods that reliably reproduce preclinical results at clinical trial. 


\section{Understand the regulations}

During the translation process, TERM researchers must address additional questions that not only satisfy basic science but also meet regulatory requirements to prime their research for translation.

\subsection{Define the product}

Region-specific regulatory bodies will have their own healthcare product definitions, which primarily differentiate between drug, medical device, advanced biologics or combination products [36]. In the United States, biologics are regulated by the Center for Biologics Evaluation and Research within the Food and Drug Administration (FDA). Within the European Medicines Agency (EMA) the equivalent is referred to as an advanced therapeutic medicinal product (ATMP) and these are regulated by the Committee for Advanced Therapies. Briefly, an ATMP is defined as a gene therapy, somatic cell therapy or tissue engineered product (EC 1394/2007). Stem cell-loaded scaffolds, drug-eluting meshes and chemicalsecreting cells all fall within the combination product category [37]. Table 1 describes product classifications as defined by regulatory agencies within Europe and USA. The initial step of classification can be challenging for TERM therapies. Their mode of action (MOA) cannot be defined as simply as traditional pharmaceuticals where MOA is a critical parameter used to determine assignment of classification. Particularly for combination products, establishing the primary MOA may be necessary for identifying appropriate approval pathways [38].

Product classification will dictate which regulatory route a product follows. Class I medical devices have shorter routes to clinic with the rigor of regulation increasing with the complexity 
of the product $[39,40]$. Further breakdown of the ATMP classification will significantly change the extent of regulation if the product meets both of the following criteria: a) 'Minimally manipulated' ATMPs undergo only non-substantial procedures such as centrifugation, cell separation and cryopreservation and b) 'homologous use' is when the ATMP is intended to perform the same function in the recipient as in the donor [41]. Identifying the regulatory classification of the product can equip researchers with a framework of standards which they can refer to during research, development and pre-clinical stages to ensure two key authorisations are obtained: firstly, achieving approval to conduct human clinical trials and secondly gaining marketing authorisation [42] (Figure 1). 


\subsection{Ensure compliance with regulatory standards}

There are good manufacturing practice (GMP) standards that can be met early in development, which will help build a robust portfolio of data to ease translation (Figure 1). Briefly discussed in Section 1.2, GLP should be exercised during early stages of translational research and beyond. GLP standards should be adhered to by any facility that carries out non-clinical safety testing with respect to human or environmental health. The standards ensure that the data gained from these studies are reliable and can be used for generating regulatory risk/safety assessments. GLP requires record keeping of lot numbers, raw data, appropriate test and control specimens, use of SOPs and evidence of robust study design. GMP is concerned with consistent production processes so that every batch of therapeutic will meet defined safety and efficacy standards. This will have a critical role at the later stages of product development where quality control becomes a requisite for market approval. Early compliance strategies to aid translation of the research include:

1. Developing SOPs for all stages of production to promote consistency and to include quality assurance testing for characterization (21 CFR 610.14 2006) and contaminants (21 CFR 610.13) $[43,44]$. Determining that the necessary standards for the product can be achieved by communication with regulatory authorities and organisations that establish standards for regulatory processes. The International Organisation for Standardisation, for example, can provide ISO standards for medical device quality management systems (ISO 13485:2003) and requirements for processing practices of medical products containing viable human cells (ISO 13022:2012). 
2. Diligent sourcing of raw materials (from a regulatory perspective). Cell therapy products may involve the use of xenogeneic components such as FBS or gelatin. These may contain contaminants that cause bovine spongiform encephalopathy (BSE), and as a result regulatory bodies will ask for these materials to be sourced from animals that reside in countries certified to be free of BSE [45]. Non-clinical-grade cell sources or risk of infectious or genetic disease transmission may present significant hurdles for translation [42, 43].

3. Consider the current approval status for all materials. When developing a new product all materials used to construct that product should be assessed. This includes all reagents that come into contact with the product during manufacture. For example, building a combination product including cells and a biomaterial will require full approval of both components.

4. Pre-clinical testing. Develop in vitro and animal models to collect data output that is relevant from a regulatory standpoint including initial safety, efficacy, purity, dose response and MOA in order to make the transition towards applying for approval as an investigational product more likely (Figure 1). 


\subsection{Identify the regulatory route to market and inform the regulators if necessary}

Traditionally, once a pharmaceutical product has been classified by regulators, it will follow a particular well-trodden route to approval for market authorisation. However, as the TERM field is still nascent, established approval routes are lacking, which opens up the opportunity to work closely with regulators to develop a suitable route for the product.

The FDA approved its first tissue engineered product, Apligraf ${ }^{\mathrm{TM}}$, in 1998. This product, containing viable human fibroblasts, was classified as a medical device and approved via the pre-market approval route for the treatment of chronic skin ulcers [46, 47]. Since then, many TERM products have emerged and may follow differing routes for approval (Table 2) due to regulatory learning and changes in the environment.

For example, an important recent discussion for the reform of TERM regulation is the introduction of adaptive licensing. Japan is overhauling the regulatory landscape by introducing two new acts: one that covers regenerative medicine therapies without market approval, such as for research purposes and private practice within medical institutions (the Act on the Safety of Regenerative Medicine). The other regulates the market approval of regenerative medicine products for wider distribution (Pharmaceutical and Medical Devices Act) [48]. The latter act proposes a fast track system to enable time-constrained approval with exploratory clinical trial data that proves safety and 'probable' benefit. Extended post-market surveillance for prospective evidence gathering is allowed with subsequent adaptation to the original marketing authorisation. This overhaul has catalysed similar developments in Canada and the EU, where "conditional approval and staggered licensing" are being considered [49]. 
Due to these many variations and continuous developments of regulatory pathways, it is encouraged to reach out to regulators at early stages to gain scientific advice. 


\section{Understand the implications for manufacture}

Identified by the UK's national stock take of regenerative medicine as a significant challenge [50], the development of scalable, reproducible processes whilst retaining the product's CQAs is critical for translational, and ultimately product success. Unlike conventional biopharmaceutical production, cells form the basis of many TERM products. Product (cell) quality must therefore be maintained all the way through from donor isolation to patient delivery.

\subsection{Find out what is relevant and measure it}

Improvement of production processes is achieved by obtaining a greater understanding of the product's functional metrics. This understanding will arise through the development of novel measurement and characterisation technologies, providing opportunities for innovation and novel technology development. The progress of non-invasive live cell imaging and its utilisation as a process analytical tool is one such example of the integration of novel technologies to improve process understanding and consistency [51].

Improved product understanding is driven by the need to maintain quality in inherently variable processes. It is imperative therefore that relevant functional metrics are identified, measured and that the range of allowable variation (as determined by preclinical and clinical trial data) is quantified, whilst maintaining product quality for each process step and ensuring that the extent of variation remains within these tolerances $[6,7]$. Furthermore, the traceability or correlation to a common reference, along with method validation, completes the verification of 
process metrology. This will drive the development of robust control systems to ensure the process always remains in a 'state of control'.

\subsection{Consistently, consistently, consistently}

In regulator Health Canada's Summary Basis of Decision for Remestemcel-L (Prochymal® an allogeneic human mesenchymal stem cell therapy for acute Graft versus Host Disease), one word reverberated throughout: "consistently" [52]. From the document's conclusions: “...the Chemistry and Manufacturing information submitted for Prochymal has demonstrated that the drug substance and drug product can be consistently manufactured to meet the approved specifications". It would be remiss of the translational researcher to ignore the fact that consistent manufacture is a prerequisite for regulatory approval. Developing consistent processes and repeatable procedures may require the adoption of chemically defined xeno-free production and implementation of methods aimed at identifying and reducing variation in biological source material. Investing in consistent manufacture and developing repeatable procedures can provide economic value, for example by reducing process deviation (and therefore the risk of batch failure) and increasing production capacity. It demonstrates a 'state of control' and a high degree of product understanding, improving the likelihood of regulatory approval.

It is also worth noting that whilst consistency is paramount to any TERM manufacturing process, achieving this can be extremely difficult, particularly for inherently variable bioprocesses such as autologous processes where donor material will differ from patient to patient [53], as opposed to allogeneic processes where there is a degree of control with respect 
to biological starting material. Whilst it is unfeasible to eliminate variation entirely, understanding the sources of variation and identifying strategies to minimise these is critical and may result in a situation where differences in the product do not affect patient outcome [53].

\subsection{Consider scale from the outset}

In the pharmaceutical/biopharmaceutical industries, efficiency and productivity typically plummet when processes are transferred from laboratory to industrial scale [54]. As part of due diligence and risk management, scalability should be considered from the outset, including an early assessment of cost of goods and supply chain risks.

To avoid technical issues associated with scale-up, small-scale models that demonstrate comparability with larger-scale systems should be adopted where possible. For example, smallscale stirred-tank bioreactor platforms are more reliable predictors of large-scale performance than traditional shake-flask models, reducing time to market and cost of goods [55]. Moreover, translational researchers should employ quality tools such as risk assessments and process maps to break down each process step to its constituent components and ensure that each process platform is scalable, and identify what process steps, if any, can be eliminated entirely or replaced with an alternative, more readily scalable step. Examples of these quality tools include FMEAs (Failure Mode and Effect Analysis), Ishikawa Diagrams and Pareto Charts [6]. The use of quality tools, for example, may identify reagents or consumables that are not suitable for scaled manufacture. As described above (Section 2.2) raw materials must be selected and sourced carefully in order to minimise variability. Where possible, alternatives to process 
critical components (i.e. surface-coatings, growth factors etc.) should be identified and if no alternative is available, the risk should be acknowledged and mitigated, for example, by securing future supply in the event of large-scale production.

Additionally, the relevant business processes should be mapped out and it should be determined which particular manufacturing processes will be constrained by economic and value-chain drivers. This may reveal insurmountable cost of goods arising from impracticable scaled production, allowing for alternative options to be considered. It is worth noting that the production and business processes will vary significantly depending on the final product. For example, a product such as autologous $\mathrm{CD} 34^{+}$cell transplantation which involves only the enrichment of a patient's cells will require a different manufacturing process to an autologous process which requires direct reprogramming and subsequent differentiation of the patient's starting material. This also has regulatory implications as discussed in Section 4.1, particularly with respect to a product's 'minimally manipulated' status. 


\section{Manage the commercial potential of the research}

Clinical translation of TERM therapies depends on successful project management to develop a product that meets both clinical need and safety and regulatory requirements. Additionally, effective IP management is vital to deliver a viable product that offers an investable proposition to those involved. Technology transfer offices (TTOs) often provide expert, free advice to ECRs on whether research is protectable by IP and how it can be translated.

\subsection{Protect the idea to establish commercial value}

IP protection secures an idea's exclusivity and indicates value to potential investors. Deciding which aspects of an idea are suitable for IP protection, and who is the rightful owner of an invention, depends on contributions to both the conception and execution of an idea, as well as contractual agreements at individual institutions (Textbox 2). To maximise the impact of research and commercial value across several fields, ECRs should identify translational IP space, and appreciate its breadth. For example, one should consider whether the concept could be developed as a platform technology, with multiple potential applications (e.g. platforms applicable to various patient-specific autologous cells) [56].

Researchers should also consider whether to seek protection in the UK, Europe, US or worldwide, and once protected, they can choose whether to self-develop (in-house or through academic/industrial collaborations), or to assign IP to another party. TTOs can assist with writing IP applications and often fund a bid for one year's protection, during which proof-ofconcept research and market value investigations can be undertaken. A full application may then be funded by the inventors, TTO, or other backers; however without funding, protection ends after one year, so sourcing funding for full IP protection is a crucial translational hurdle. 
Academic ECRs should also consider the increasing pressure to disseminate research and offset this with the consequences of invalidating IP by revealing prior art. For collaborative research, describing information as proprietary or signing confidential disclosure agreements (CDAs) can safeguard ideas prior to IP applications.

[Textbox 2]

\section{Is it patentable?}

To be patentable, ideas must be novel, not obvious, and useful [57]. This could include a new use for a pre-existing idea.

\section{Who is the inventor?}

The person conceiving the idea, or a novel step in the fabrication of the idea [57]. People instructed to perform experiments/research/development are not inventors; they have "reduced to practice" a novel idea. The inventor is often not the owner of the idea; the employer is usually the owner.

\subsection{Market the concept and develop a network}

Successful project management (described in Section 1) should allow ECRs to identify, liaise and engage with appropriate bodies to develop a translational pathway. This may involve forming a 'spin out' or joint venture, which allows the inventor to retain some control over IP commercialization. Alternatively, IP can be sold or licenced to third parties through technology licencing agreements; however, the inventor's involvement usually ends here. Building a personal network with those in relevant fields will enable both the development of one's reputation and allow for the identification of collaborators who can aid translation. Forming 
collaborations with those experienced in scale up, automation or other manufacturing issues can help accelerate the transition to market. Importantly, national and international consortia facilitating translation from research concept to commercialisation through collaborations are becoming more common, providing straightforward access to a range of expertise $[58,59]$.

\subsection{Leverage funding}

Funding will be required throughout stages of the translation process (Figure 3). Research councils and charities commonly fund proof-of-concept research, but have varying eligibility regarding funds for later stage commercialisation such as Phase I through Phase III trials [60]. Instead, alternative funding sources such as business angels, key opinion leaders or commercialisation seed funding schemes can be accessed for support (Table 3). Eligibility criteria for these highly competitive schemes vary, often requiring matched funding from an industrial collaborator (which can be limited to small/medium enterprises, SMEs), but funders may offer additional support during the application process and post-award. Preparing bids is time-consuming, so try to get early notice via automated notifications/alerts (e.g. Google alerts, mailing lists) and leverage collaborators' knowledge of funding trends and requirements to save both time and money. 


\section{Conclusion}

Having strategically designed the concept with clinical relevance, manufacturing and regulatory hurdles in mind, validated the scientific worth, protected the idea, and sourced appropriate funding and collaborators, translation into a commercial product is feasible. Although navigating through the translational landscape may seem daunting, we suggest that ECRs can increase their chances of success by tactically planning their research to address these common pitfalls early, minimising the chances of having to reiterate a design step later on in the commercialisation process.

\section{Future perspective}

Clinical and commercial failures in recent years have reinforced the need for the translation of TERM therapies. The industry is therefore entering a critical phase which can no longer rely on potential, but is expected to deliver efficacious, cost-effective products. Whilst this presents a significant challenge, the TERM industry is ideally positioned to capitalise on recent clinical success (e.g. T-cell immunotherapy) amidst a backdrop of renewed government/state investment (e.g. Cell Therapy Catapult, Centre for Commercialization of Regenerative Medicine). What imbues the greatest confidence of success, however, is the emergence of a generation of highly trained, committed, translationally-focussed ECRs.

Although faced with an uncertain regulatory/reimbursement landscape and complex scientific/technical obstacles, global recognition of the importance of translation will ensure that translational ECRs are given the appropriate authority to play a central role in the realisation of TERM therapies. We believe translational ECRs will rise to the challenge, 
learning from mistakes of the past, adopting best practices from closely aligned industries and developing innovative solutions to TERM-specific issues in a pre-competitive environment.

\section{Executive summary}

As translational ECRs in TERM, we have come to realise that the process of translation can be intricate, exceptionally resource consuming and prone to hidden obstacles. We have therefore compiled 'tips and tricks', based on our own experience and research that facilitate translation and will improve the chances of successful product development.

\section{Manage the research effectively}

- Utilise project management tools and divide the research into work packages with clear milestones and deliverables. Effective research management also includes day-to-day planning, mapping out the experimental process and identifying high risk areas and ways of mitigating these.

\section{Ensure the data are scientifically robust}

- Experiments in TERM are resource heavy. Take time and care in planning experiments and implement systematic methods such as Design of Experiments. Source raw materials carefully and ensure that the principles of any technology, assay or technique are well understood to ensure maximum output of reliable and robust data.

\section{Ensure the work has clinical relevance}

- To increase chances of clinical and commercial success, research should be aligned with appropriate clinical pull, and where possible developed as platform technologies. 
In addition, many current assays are unreliable, or unsuitable for testing TERM therapies, but these shortcomings provide rich opportunities for the development of new, TERM-relevant models with genuine predictive utility.

\section{Understand the regulations}

- Identify early on in the research programme the regulatory classification of the potential product to provide a framework of standards which can be referred to. Build compliance of regulatory standards into the process, and when necessary, be prepared to engage and inform the regulator.

\section{Understand the implications for manufacture}

- Relevant functional metrics, with associated tolerances, need to be identified and quantified to improve process and product understanding. The pursuit of consistency in manufacture is paramount and efforts must be made to avoid or minimise sources of variation. Scalability should be considered from the outset with a focus not only on the technical challenges but also cost of goods and supply chain issues associated with scaled production.

\section{Commercialise the research}

- IP protection is generally necessary to demonstrate commercial value. Effective partnerships and collaborations will accelerate commercialisation and de-risk the venture for potential investors and funders to invest. Develop an effective funding strategy, stratifying funding sources if necessary and leveraging funds from one source with another. 


\section{Acknowledgements}

The authors would like to thank Professors David Williams (Loughborough University, UK) and John Fisher (University of Leeds, UK) for their guidance and direction in the preparation of this manuscript.

\section{Financial \& competing interests disclosure}

The authors would like to acknowledge the support of the Engineering and Physical Sciences Research Council for the funding of the Engineering, Tissue Engineering and Regenerative Medicine (E-TERM) Landscape Fellowship programme, which involves a partnership of Loughborough, Leeds, Sheffield, Keele, Nottingham and York Universities. All authors, with the exception of Dr David Smith are current E-TERM Fellows or E-TERM alumni. Dr David Smith is a Biomedical Engineer at PCT (a Caladrius Company). The authors have no other relevant affiliations or financial involvement with any organization or entity with a financial interest in or financial conflict with the subject matter discussed in the manuscript apart from those disclosed. No writing assistance was utilized in the production of this manuscript. 


\section{References}

1. Cyranoski D: Collateral damage: How a case of misconduct brought a leading Japanese biology institute to its knees. Nature 520(7549), 600-603 (2015).

2. $\quad$ Cyranoski D: Whistle-blower breaks his silence. Nature 505(7485), 593-594 (2014).

3. Fogler HS: Experimental planning. In: Essentials of chemical reaction engineering, (Ed.^(Eds). Pearson Education, Inc, NJ, USA 271-272 (2011).

4. Kirouac DC, Zandstra PW: The systematic production of cells for cell therapies. Cell Stem Cell 3(4), 369-381 (2008).

5. Placzek MR, Chung IM, Macedo HM et al:: Stem cell bioprocessing: fundamentals and principles. J. R. Soc. Interface 6(32), 209-232 (2009).

6. Williams DJ, Thomas RJ, Hourd PC et al.: Precision manufacturing for clinicalquality regenerative medicines. Philos Trans A Math Phys Eng Sci 370(1973), 39243949 (2012).

7. Ratcliffe E, Thomas RJ, Williams DJ: Current understanding and challenges in bioprocessing of stem cell-based therapies for regenerative medicine. $\mathrm{Br}$. Med. Bull. 100(1), 137-155 (2011).

8. Bravery CA, Carmen J, Fong $T$ et al:: Potency assay development for cellular therapy products: an ISCT review of the requirements and experiences in the industry. Cytotherapy 15(1), 9-19 (2013).

9. Burger SR, Juliano L, Wang W: Cellular raw material collection in cell therapy. Drug Discovery World Summer 2014, 29-34 (2014).

10. Lionberger RA, Lee SL, Lee L, Raw A, Yu LX: Quality by Design: Concepts for ANDAs. The AAPS Journal 10(2), 268-276 (2008).

11. Zimmerman RL: 10 best practices of good laboratories. ASTM Stand. News 38(6), 24-31 (2010).

12. Brosnan K, Want A, Coopman K, Hewitt CJ: Multiparameter flow cytometry for the characterization of human embryonic stem cells. Biotechnol. Lett. 35(1), 55-65 (2013).

13. Shapiro HM: Practical flow cytometry. John Wiley \& Sons, (2005).

14. Plagnol AC, Rowley E, Martin P, Livesey F: Industry perceptions of barriers to commercialization of regenerative medicine products in the UK. Regen. Med. 4(4), 549-559 (2009).

15. Couto DS, Perez-Breva L, Cooney CL: Regenerative medicine: learning from past examples. Tissue Eng. Part A 18(21-22), 2386-2393 (2012).

16. Cai M, Yang Y: Targeted genome editing tools for disease modeling and gene therapy. Curr. Gene Ther. 14(1), 2-9 (2014).

17. Mason C: Regenerative medicine 2.0. Regen. Med. 2(1), 11-18 (2007).

18. Bubela T, Mccabe C: Value-engineered translation for regenerative medicine: meeting the needs of health systems. Stem Cells Dev. 22 Suppl 1, 89-93 (2013).

19. Bertram TA, Tentoff E, Johnson PC, Tawil B, Van Dyke M, Hellman KB: Hurdles in tissue engineering/regenerative medicine product commercialization: a pilot survey of governmental funding agencies and the financial industry. Tissue Eng. Part A 18(21-22), 2187-2194 (2012).

20. Weinstein MC, Torrance G, Mcguire A: QALYs: The Basics. Value Health 12, S5-S9 (2009). 
21. Wouters S, Van Exel NJA, Rohde KIM, Brouwer WBF: Are all health gains equally important? An exploration of acceptable health as a reference point in health care priority setting. Health Qual. Life Outcomes 13(1), 1-10 (2015).

22. Brinker M, O'connor D: Stakeholders in Outcome Measures: Review From a Clinical Perspective. Clin Orthop Relat Res 471(11), 3426-3436 (2013).

23. Mcateer H, Cosh E, Freeman G, Pandit A, Wood P, Lilford R: Costeffectiveness analysis at the development phase of a potential health technology: examples based on tissue engineering of bladder and urethra. J. Tissue Eng. Regen. Med. 1(5), 343-349 (2007).

24. Trounson A, Baum E, Gibbons D, Tekamp-Olson P: Developing a case study model for successful translation of stem cell therapies. Cell Stem Cell 6(6), 513-516 (2010).

25. Rose JB, May M, Williams DJ: Determinants of clinician adoption of regenerative therapies in the UK and Canada: an ophthalmology perspective. Regen. Med., 1-13 (2015).

26. Champkin J: George E. P. Box, 1919-2013. Significance 10(3), 32-34 (2013).

27. Box GEP: In: Robustness in Statistics, Launer RL,Vilkinson GN (Ed.^(Eds). Academic Press, 201-236 (1979).

28. Leist M, Hartung T: Inflammatory findings on species extrapolations: humans are definitely no 70-kg mice. Arch. Toxicol. 87(4), 563-567 (2013).

29. Lemmon VP, Ferguson AR, Popovich PG et al.: Minimum information about a spinal cord injury experiment: a proposed reporting standard for spinal cord injury experiments. J. Neurotrauma 31(15), 1354-1361 (2014).

30. Lowenstein PR, Castro MG: Uncertainty in the translation of preclinical experiments to clinical trials. Why do most phase III clinical trials fail? Curr. Gene Ther. 9(5), 368-374 (2009).

31. Cook N, Jodrell DI, Tuveson DA: Predictive in vivo animal models and translation to clinical trials. Drug Discov. Today 17(5-6), 253-260 (2012).

32. Andersen ME, Betts K, Dragan $\mathrm{Y}$ et al: Developing microphysiological systems for use as regulatory tools--challenges and opportunities. Altex 31(3), 364-367 (2014).

33. Balls M: The origins and early days of the Three Rs concept. Alternatives to laboratory animals : ATLA 37(3), 255-265 (2009).

34. Liebsch $M$, Grune B, Seiler A et al.: Alternatives to animal testing: current status and future perspectives. Arch. Toxicol. 85(8), 841-858 (2011).

35. Varga OE, Hansen AK, Sandoe P, Olsson IA: Validating animal models for preclinical research: a scientific and ethical discussion. Alternatives to laboratory animals : ATLA 38(3), 245-248 (2010).

36. Fda: Guidance for industry and FDA staff: classification of products as drugs and devices and additional product classification issues. 2015(April), (2011).

37. Lee JK, Responte DJ, Cissell DD, Hu JC, Nolta JA, Athanasiou KA: Clinical translation of stem cells: insight for cartilage therapies. Crit. Rev. Biotechnol. 34(1), 89-100 (2014).

38. Fda: Definition of primary mode of action of a combination product. Final rule. Fed. Regist. 70(164), 49848-49862 (2005).

39. Kramer DB, Xu S, Kesselheim AS: Regulation of Medical Devices in the United States and European Union. N. Engl. J. Med. 366(9), 848-855 (2012).

40. Pashuck ET, Stevens MM: Designing regenerative biomaterial therapies for the clinic. Science translational medicine 4(160), 160sr164 (2012). 
41. European Medicines Agency, Committee for Advanced Therapies (Cat): Reflection paper on classification of advanced therapy medicinal products (EMA/ CAT/600280/2010) (2012).

42. Baum E, Littman N, Ruffin M, Ward S, Aschheim K: White paper: Key tools and technology hurdles in advancing stem-cell therapies. 121, (2013).

43. Halme DG, Kessler DA: FDA Regulation of Stem-Cell-Based Therapies. $N$. Engl. J. Med. 355(16), 1730-1735 (2006).

44. Prestwich GD, Bhatia S, Breuer CK et al.: What is the greatest regulatory challenge in the translation of biomaterials to the clinic? Science translational medicine 4(160), 160cm114 (2012).

45. Fda: Medical Devices Containing Materials Derived from Animal Sources (Except for In Vitro Diagnostic Devices) 2015(April), (1998).

46. Kemp P: History of regenerative medicine: looking backwards to move forwards. Regen. Med. 1(5), 653-669 (2006).

47. Fda: Summary of safety and effectiveness data for Apligraf ${ }^{\mathrm{TM}}$. (1998).

48. Azuma K: Regulatory Landscape of Regenerative Medicine in Japan. Curr Stem Cell Rep, 1-11 (2015).

49. Cyranoski D: Japan to offer fast-track approval path for stem cell therapies. Nat. Med. 19(5), 510-510 (2013).

50. Office for Life Sciences: Taking stock of regenerative medicine in the United Kingdom. (2011).

51. Narkilahti S, Rajala K, Pihlajamaki H, Suuronen R, Hovatta O, Skottman H: Monitoring and analysis of dynamic growth of human embryonic stem cells: comparison of automated instrumentation and conventional culturing methods. Biomed. Eng. Online 6, 11 (2007).

52. Health Canada: Summary Basis of Decision (SBD) for PROCHYMAL $®$. 2012(15th October), (2012).

53. Thurman-Newell JA, Petzing JN, Williams DJ: Quantification of biological variation in blood-based therapy - a summary of a meta-analysis to inform manufacturing in the clinic. Vox Sang., n/a-n/a (2015).

54. Rafiq QA, Hewitt CJ: Cell therapies: why scale matters. Pharm. Bioprocess. 3(2), 97-99 (2015).

55. Pollard D: Are automated disposable small-scale reactors set to dominate the future of pharmaceutical bioprocess development? Pharm. Bioprocess. 2(1), 9-12 (2014).

56. Fierabracci A: Recents Patents for Isolating, Delivering and Tracking Adult Stem Cells in Regenerative Medicine. Recent Pat. Drug Delivery Formulation 4(2), 105-113 (2010).

57. Ogl: Intellectual property and your work. 2015(March), (2014).

58. Willemse L, Lyall D, Rudnicki M: Catalyzing stem cell research. Regen. Med. 3(5), 761-764 (2008).

59. Munisi HI, Xie Z, Sengoku S: Exploring innovation in stem cell and regenerative medicine in Japan: the power of the consortium-based approach. Regen. Med. 9(4), 467-477 (2014).

60. Research Councils Uk: Material world: knowledge economy. 2015(April), (2004).

61. British Standards Institute (Bsi): PAS83:2012 Developing human cells for clinical applications in the European Union and the United States of America. (2012). 


\section{Reference annotations}

* Lowenstein PR, Castro MG. Uncertainty in the translation of preclinical experiments to clinical trials. Why do most phase iii clinical trials fail? Curr. Gene Ther. 9(5), 368-374 (2009).

An easily-digestible survey of why clinical failures occur, with an important rallying call for 'preclinical robustness'.

* Weinstein MC, Torrance G, Mcguire A. Qalys: The basics. Value Health 12, S5-S9 (2009).

A review of the concept of quality-adjusted life year, its evolution and the proposal of alternative conceptual models for measuring health and cost-effectiveness.

* Varga OE, Hansen AK, Sandoe P, Olsson IA. Validating animal models for preclinical research: A scientific and ethical discussion. Alternatives to laboratory animals : ATLA 38(3), 245-248 (2010). A comprehensive overview of the urgent need for, and a proposed route towards, preclinical model validation.

* Bravery CA, Carmen J, Fong T et al. Potency assay development for cellular therapy products: An ISCT review of the requirements and experiences in the industry. Cytotherapy 15(1), 9-19 (2013). A comprehensive review of the requirements for defining and measuring the quality of cellular products, a prerequisite for successful translation.

* Rafiq QA, Hewitt CJ. Cell therapies: why scale matters. Pharmaceutical Bioprocessing 3(2), 97-99 (2015). 
A perspective on the importance of understanding the significance and impact of scale for therapeutic bioprocess development.

\section{References of considerable interest}

** Williams DJ, Thomas RJ, Hourd PC et al. Precision manufacturing for clinical-quality regenerative medicines. Philosophical Transactions. Series A, Mathematical, Physical, and Engineering Sciences 370(1973), 3924-3949 (2012).

An overview of the importance precision manufacturing in the TERM field with a description of quality engineering techniques and their application for successful translation.

** Azuma K. Regulatory landscape of regenerative medicine in Japan. Curr Stem Cell Rep, 1-11 (2015) A brief but important paper providing an overview of the regulatory, reimbursement and other strategic changes in Japan with respect to regenerative medicine. 


\begin{tabular}{|c|c|c|c|}
\hline & Definition* (EU/USA) & $\begin{array}{c}\text { Regulatory } \\
\text { Agency } \\
\text { Within EMA/ } \\
\text { FDA }\end{array}$ \\
\hline \multirow{3}{*}{ 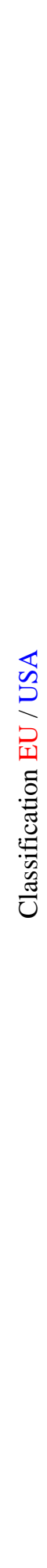 } & 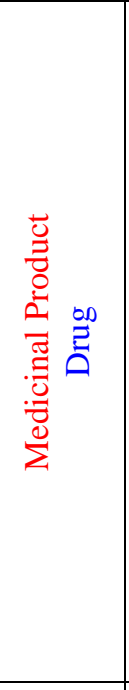 & $\begin{array}{l}\text { Any substance or combination of substances presented as having } \\
\text { properties for treating or preventing disease in human beings; or } \\
\text { b) Any substance or combination of substances which may be } \\
\text { used in or administered to human beings either with a view to } \\
\text { restoring, correcting or modifying physiological functions by } \\
\text { exerting a pharmacological, immunological or metabolic action, } \\
\text { or to making a medical diagnosis. [Directive 2001/83/EC, Article } \\
\text { 1.2] } \\
\text { a) Articles recognized in the official United States } \\
\text { Pharmacopoeia, official Homoeopathic Pharmacopoeia of the } \\
\text { United States, or official national formulary, or any supplement } \\
\text { to any of them; and b) articles intended for use in the diagnosis, } \\
\text { cure, mitigation, treatment, or prevention of disease in man or } \\
\text { other animals; and c) articles (other than food) intended to affect } \\
\text { the structure or any function of the body of man or other animals; } \\
\text { and d) articles intended for use as a component of any article } \\
\text { specified in Clause a), b), or c). [21 USC 321(g)(1)] }\end{array}$ & $\begin{array}{l}\text { Committee for } \\
\text { Medicinal } \\
\text { Products for } \\
\text { Human use } \\
\text { (CHMP) }\end{array}$ \\
\hline & 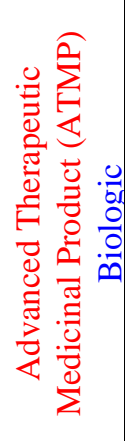 & $\begin{array}{l}\text { Consists of either: A gene therapy medicinal product as defined } \\
\text { in Part IV of Annex I to Directive 2001/83/EC, - a somatic cell } \\
\text { therapy medicinal product as defined in Part IV of Annex I to } \\
\text { Directive 2001/83/EC, - a tissue engineered product as defined } \\
\text { in (1(d) (EC) No. 1394/2007). } \\
\text { Biological products include vaccines, blood and blood } \\
\text { components, allergenics, somatic cells, gene therapy, tissues, and } \\
\text { recombinant therapeutic proteins. Biologics can be composed of } \\
\text { sugars, proteins, or nucleic acids or complex combinations of } \\
\text { these substances, or may be living entities such as cells and } \\
\text { tissues. }\end{array}$ & $\begin{array}{l}\text { Committee for } \\
\text { Advanced } \\
\text { Therapies } \\
\text { (CAT) } \\
\text { Center for } \\
\text { Biologics } \\
\text { Evaluation and } \\
\text { Research } \\
\text { (CBER) }\end{array}$ \\
\hline & 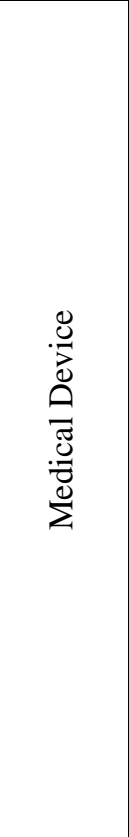 & $\begin{array}{l}\text { Any instrument or other article to be used in human beings for } \\
\text { the purpose of: } \\
\text { a) diagnosis, prevention, monitoring, treatment or alleviation of } \\
\text { disease or compensation for an injury or handicap, b) } \\
\text { investigation, replacement or modification of the anatomy or of a } \\
\text { physiological process, c) control of conception, and which does } \\
\text { not achieve its principal intended action in or on the human body } \\
\text { by pharmacological, immunological or metabolic means, but } \\
\text { which may be assisted in its function by such means. [Directive } \\
\text { 93/42/EEC, article 1.2(a)] 90/385/EEC } \\
\text { An instrument or related article which is: } \\
\text { - intended for use in the diagnosis of disease or other conditions, } \\
\text { or in the cure, mitigation, treatment, or prevention of disease, in } \\
\text { man or other animals, or } \\
\text { - intended to affect the structure or any function of the body of } \\
\text { man or other animals, and which does not achieve its primary } \\
\text { intended purposes through chemical action within or on the body } \\
\text { of man or other animals and which is not dependent upon being } \\
\text { metabolized for the achievement of any of its primary intended } \\
\text { purposes." } \\
21 \text { USC } 321(\mathrm{~h})\end{array}$ & $\begin{array}{l}\text { Notified Body } \\
\text { (NB) } \\
\text { Center for } \\
\text { Devices and } \\
\text { Radiological } \\
\text { Health (CDRH) }\end{array}$ \\
\hline
\end{tabular}




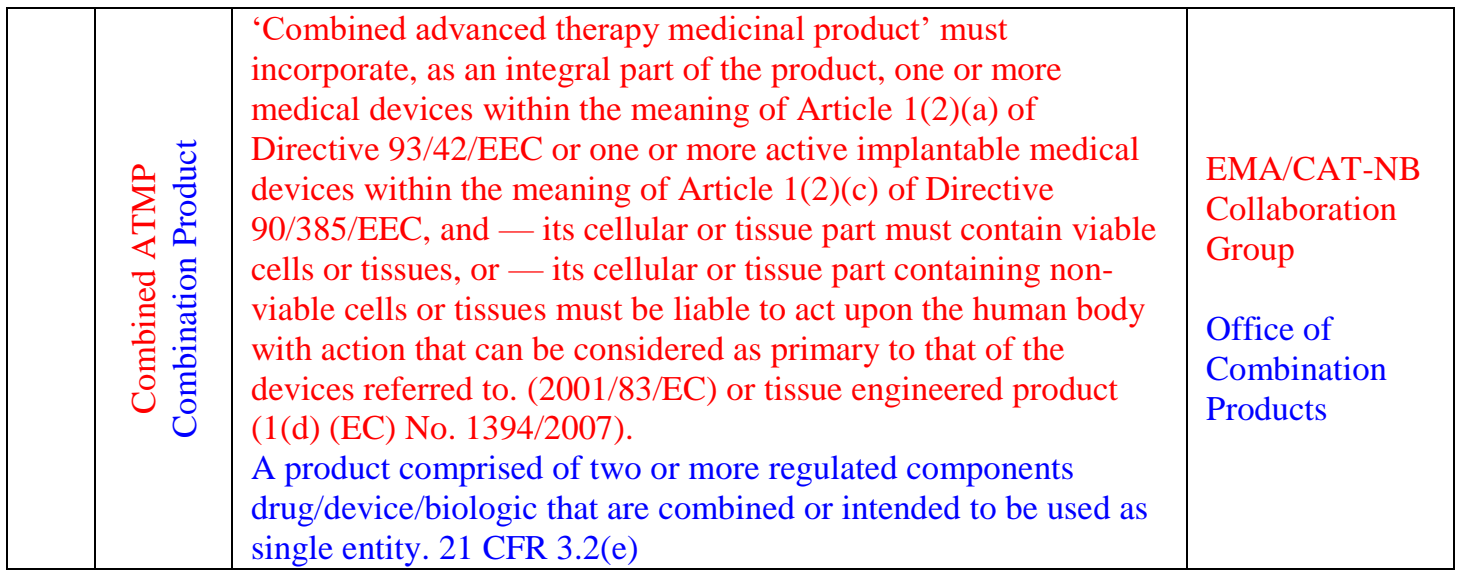

Table 1. Healthcare product definitions. Classifications for healthcare products are listed here as described by regulatory bodies within Europe (EMA, red) and USA (FDA, blue). Adapted from [61]. *For complete definitions refer to the associated regulatory directive. 
Table 2. Regulatory routes for selected TERM products from the European and American market.

\begin{tabular}{|c|c|c|}
\hline Product & Description & Regulatory Route \\
\hline Prochymal & $\begin{array}{l}\text { Human mesenchymal stem cells } \\
\text { for treatment of GVHD. }\end{array}$ & $\begin{array}{l}\text { Conditional Approval (Canada, 2012) } \\
\text { Expanded Access (USA, 2008) }\end{array}$ \\
\hline $\begin{array}{l}\text { Holoclar } \\
\text { (EMA/786996/2014) }\end{array}$ & $\begin{array}{l}\text { Limbal epithelial stem cell } \\
\text { therapy }\end{array}$ & Conditional Approval (EU, 2015) \\
\hline OP-1 Putty & $\begin{array}{l}\text { Bovine collagen graft with } \\
\text { rhBMP-7 }\end{array}$ & $\begin{array}{l}\text { Humanitarian Device Exemption } \\
\text { (USA, 2004) }\end{array}$ \\
\hline Carticel & $\begin{array}{l}\text { Autologous cultured } \\
\text { chondrocytes for cartilage defects }\end{array}$ & Biologics license (USA, 1997) \\
\hline MySkin & $\begin{array}{l}\text { Autologous human keratinocytes } \\
\text { for burns }\end{array}$ & $\begin{array}{l}\text { Unlicensed medicinal product (UK, } \\
\text { 2004) }\end{array}$ \\
\hline
\end{tabular}


Table 3. Examples of funding sources used to commercialise research. Funders are categorised based on who the funds are linked to, the location of funded research, the type of application process, the value of the award and the phase of the translational pathway that the award funds.

\begin{tabular}{|c|c|c|c|c|c|c|c|c|c|c|c|c|c|c|c|}
\hline \multirow[t]{2}{*}{ Funding source } & \multicolumn{4}{|c|}{$\begin{array}{l}\text { Who is it linked } \\
\text { to? }\end{array}$} & \multicolumn{3}{|c|}{$\begin{array}{l}\text { Where is it } \\
\text { based? }\end{array}$} & \multicolumn{3}{|c|}{$\begin{array}{l}\text { How to } \\
\text { apply }\end{array}$} & \multirow[t]{2}{*}{ Value } & \multicolumn{3}{|c|}{ Project stage } & \multirow[t]{2}{*}{ Notes } \\
\hline & $\begin{array}{l}\frac{\pi}{E} \\
\frac{0}{0} \\
\frac{\pi}{0} \\
\frac{\pi}{\alpha}\end{array}$ & $\begin{array}{l}\frac{Z}{ \pm} \\
\stackrel{n}{D} \\
\underline{D} \\
\underline{=}\end{array}$ & 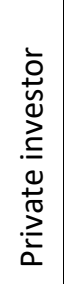 & 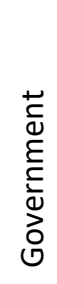 & 弚 & $\overrightarrow{\mathrm{u}}$ & $\begin{array}{l}0 \\
\stackrel{2}{\prime} \\
\vdots \\
3\end{array}$ & 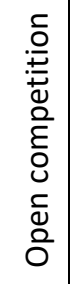 & 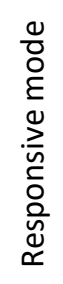 & 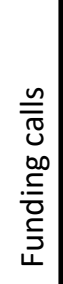 & & $\begin{array}{l}+0 \\
0 \\
0 \\
0 \\
0 \\
0 \\
4 \\
0 \\
4 \\
4 \\
0 \\
0\end{array}$ & 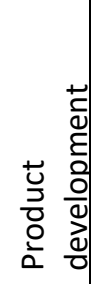 & 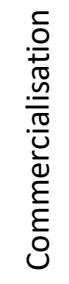 & \\
\hline Research Councils & & & & & & & & & & & Varies & & & $*$ & Individual research councils have own eligibility/criteria \\
\hline Therapy area charities & & & & & & & & & & & Varies & & & & Individual therapy area charities have own eligibility criteria \\
\hline $\begin{array}{l}\text { National competitions / } \\
\text { Entrepreneur schemes (e.g. } \\
\text { Biotechnology YES) }\end{array}$ & & & & & & & & & & & $£ 2,500$ & & & & Eligibility: Non business ECR team \\
\hline Cell Therapy Catapult & & & & & & & & & & & Varies, facilities & & & & \\
\hline Impact acceleration & & & & & & & & & & & Varies & & & & $\begin{array}{l}\text { Usually PI must have existing grant, linked to host } \\
\text { institution }\end{array}$ \\
\hline Innovate UK (formally TSB) & & & & & & & & & & & $\begin{array}{l}f 5,000-f 10 \\
\text { million }\end{array}$ & & & & Eligibility: must be UK based company linked \\
\hline Venture capital & & & & & & & & & & & Varies & & & & Requires contractual agreement with set milestones \\
\hline $\begin{array}{l}\text { Centre for Commercialization of } \\
\text { Regenerative Medicine (CCRM) }\end{array}$ & & & & & & & & & & & Facility based & & & & Based in Canada, eligibility varies \\
\hline $\begin{array}{l}\text { California Institute for } \\
\text { Regenerative Medicine (CIRM) }\end{array}$ & & & & & & & & & & & Varies & & & & Must be linked to California, awards over $\$ 10$ million \\
\hline Citizens' Innovation Fund & & & & & & & & & & & Varies & & & & $\begin{array}{l}\text { Currently limited to France, } £ 15,000 \text { per person tax free } \\
\text { general public investment in scheme }\end{array}$ \\
\hline Horizon 2020 & & & & & & & & & & & Varies & & & & Linked to academic institution \\
\hline
\end{tabular}




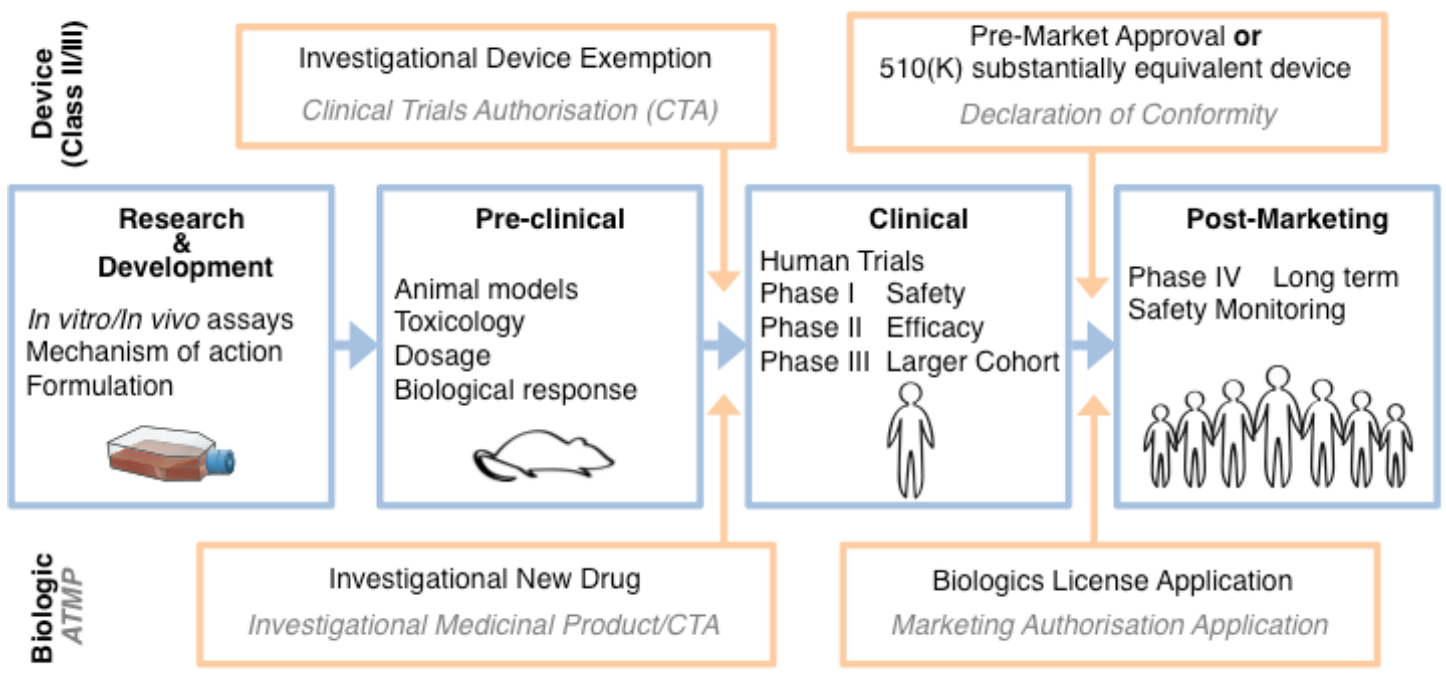

\begin{tabular}{|llll|}
\hline \multicolumn{1}{|c|}{ Regulatory Standards } & & & \\
\hline Donation \& Procurement & Good Lab Practice & Good Clinical Practice & Good Manufacturing Practice \\
21 CFR 1271 & 21CFR 58 & 21 CFR 601 & 21 CFR 210 \\
2004/23/EC & 2004/9/EC & 2009/120/EC & 2003/94/EC \\
\hline
\end{tabular}

Figure 1. Overview of regulatory approvals and standards along the pathway to translation in Europe and USA. A general comparison of the regulatory process for a medical device and a biologic/advanced therapy medicinal product in Europe (grey, italics) and the USA. Regulatory standards to be met can be found in the example directives listed. The changing regulatory landscape for TERM therapies can include other routes not depicted here such as fast track pathways that have recently been unveiled in Japan (see Section 4.3). 


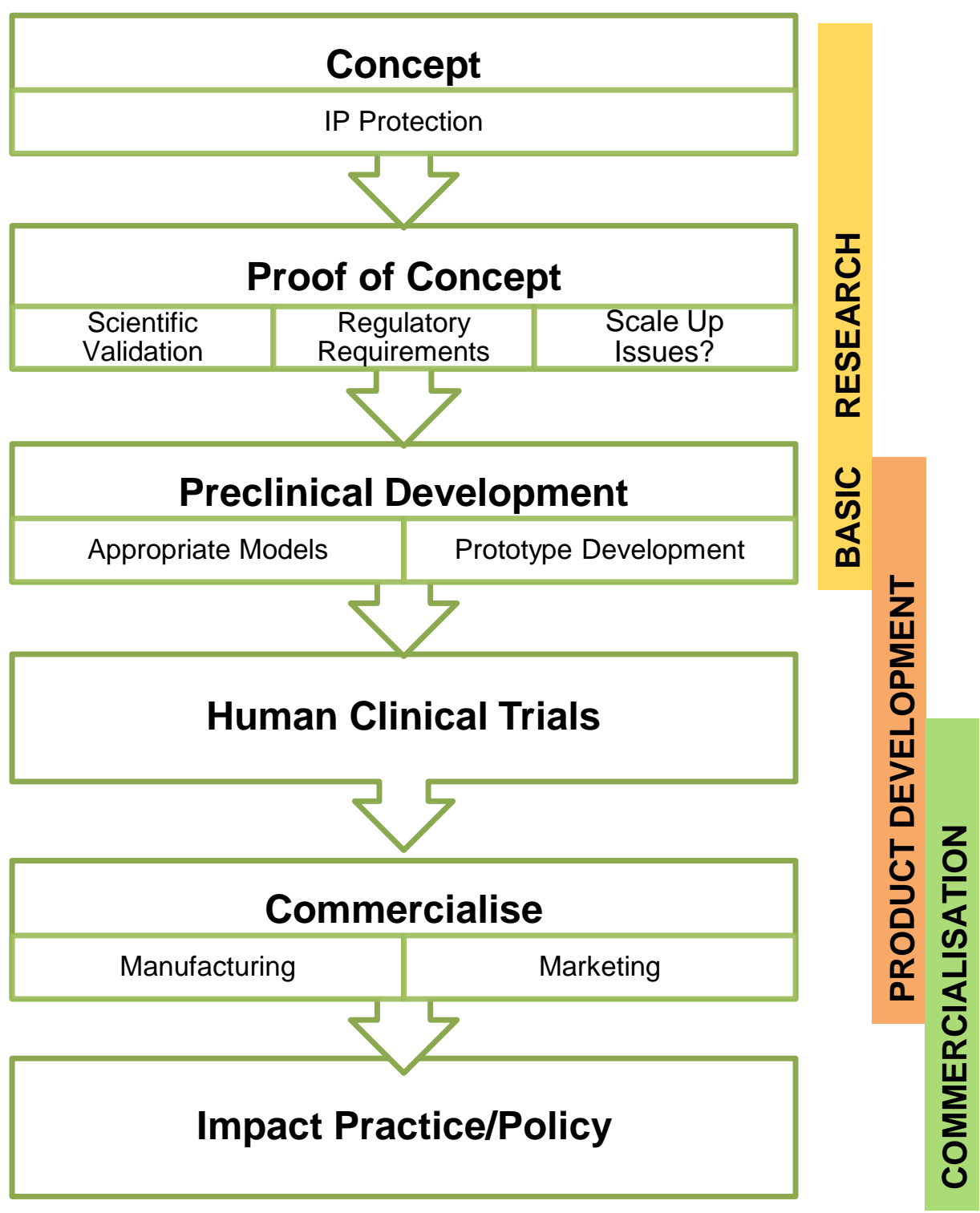

Figure 2: Translational Research Pipeline. This schematic depicts funding stages throughout translation and associated issues to consider when designing research at each stage. 